\title{
BMJ Open Mapping the links between climate change and human health in urban areas: how is research conducted? A Scoping review protocol
}

\author{
Hiago Pereira Barbosa (10 , ${ }^{1}$ Anne Roué-Le Gall, ${ }^{2,3}$ Clément Deloly, ${ }^{3}$ \\ Jean-Philippe Regnaux, ${ }^{4,5}$ Marie-Florence Thomas ${ }^{1}$
}

To cite: Barbosa HP, RouéLe Gall A, Deloly C, et al. Mapping the links between climate change and human health in urban areas: how is research conducted? A Scoping review protocol. BMJ Open 2020;10:e034667. doi:10.1136/ bmjopen-2019-034667

- Prepublication history for this paper is available online. To view these files, please visit the journal online (http://dx.doi. org/10.1136/bmjopen-2019034667).

Received 02 0ctober 2019 Revised 06 August 2020 Accepted 15 August 2020

\section{Check for updates}

(c) Author(s) (or their employer(s)) 2020. Re-use permitted under CC BY-NC. No commercial re-use. See rights and permissions. Published by BMJ.

${ }^{1}$ Université de Rennes, EHESP, Inserm, Irset (Research Institute for Environmental and Occupational Health), UMR_S 1085, Rennes, France ${ }^{2}$ Université de Rennes, EHESP, CNRS, ARENES, UMR 6051,

F-35000 Rennes, France

${ }^{3}$ Department of Environmental and occupational Health, EHESP Rennes, France

${ }^{4}$ INSERM 1153, CRESS,

EpiAgeing Team, Université de Paris, Paris, France

${ }^{5}$ Department of Social Sciences and Health, EHESP, Rennes, France

\section{Correspondence to}

Marie-Florence Thomas;

marie-florence.thomas@ehesp. fr

\section{ABSTRACT}

Introduction Scientists from a wide variety of fields of knowledge are increasingly interested in climate change issues. The importance given to the phenomenon is explained by the uncertainties surrounding it and its consequences not yet fully known. However, there is wide agreement that human activities are modifying the Earth's climate beyond the natural cyclical changes and that these changes impact human health. This scoping review aimed to understand how research on the links between climate change and human health in urban areas is conducted and how this research is approached holistically or not. Methods and analysis This scoping review is mainly guided by the Arskey and 0'Malley scoping review framework. A broad range of databases will be used, including PubMed, ScienceDirect, Web of Science Core Collection, GreenFILE and Information Science \& Technology Abstracts. Predefined inclusion and exclusion criteria will be used, with a focus on climate change and human health outcome studies published between January 1990 and July 2019. An interdisciplinary team has formulated search strategies and the reviewers will independently screen eligible studies for final study selection. We will apply a thematic analysis to evaluate and categorise the study findings. We expect to map the research according to the scientific research methods, the scientific fields and the determinants of health studied. Along these lines, we will be able to understand how holistic the research is.

Ethics and dissemination No primary data will be collected since all data presented in this review are based on published articles and publicly available documents. Therefore, ethics committee approval is not a requirement. The findings will be disseminated through publication in a peer-reviewed journal, presentations at conferences relevant to the field of this research, as well as presentations to relevant stakeholders.

\section{BACKGROUND}

Climate change is considered to be one of the main observable trends in anthropogenic effects on the global environment and human health. ${ }^{1}$ Population growth, associated with large unsustainable consumption of energy, food and other commodities,
Strengths and limitations of this study

- The first review to address the holistic approach of studies dedicated to climate change and human health.

- A study in line with the planetary health vision to move forward more integrative approaches in environmental and human health fields of development.

- Limitations related to the very limited existence of frameworks or points of reference to address the holistic approach

- The intentional choice to limit the search strategy based on two keywords-climate change and health in the titles - may lead to a bias in the analysis of the findings.

- The studies included in the scoping review will not be assessed for methodological quality.

contributes to the degradation of natural ecosystems and the worsening of health inequalities. ${ }^{2-4}$ Today, more than half of the world's population lives in urban areas, a proportion that is expected to rise to $68 \%$ by $2050 .^{5}$ In urban areas, the effects of climate change are reflected in a heterogeneous range of environmental and health consequences, such as increased heat waves, severe weather events, floods, droughts, air pollution peaks, fuel poverty and urban heat islands, among others, most of which are exacerbated by the decline of urban green spaces. ${ }^{6}$ However, most informal urban settlements is exacerbated by overcrowded and very poor-quality housing, lack of safe drinking water and easily accessible toilets, lack of sewage treatment, inadequate healthcare, lack of emergency services and other social factors. ${ }^{7-10}$

\section{Climate change: serious threat to health}

In the Fifth Assessment Report of United Nations Intergovernmental Panel on Climate Change (IPCC), climate change is defined as 
a change in the state of the climate that can be identified by modifications in the mean and/or the variability of its properties, and that persists over a long period of time. ${ }^{11}$ Climate change, whether due to natural causes (internal processes or external forcings, eg, variations of the solar cycles and volcanic eruptions) or human activities (eg, modifications in the composition of the Earth atmosphere or land use), represents a serious threat to the global population, especially to health. ${ }^{11-13}$

\section{Impacts of climate change on human health}

There is growing evidence on links between climate change and health. The adverse impacts of climate change on human health may be direct (eg, heat waves and extreme weather events such as storms, floods and droughts) or indirect (eg, degradation of water quality, air quality, land-use change, ecological change and food insecurity). ${ }^{14-16}$ These events have a potential impact on physical and mental health, as well as the well-being of the population. ${ }^{17-19}$ Children, the elderly, people with chronic diseases, pregnant women and, especially, the population living in deprived areas of urban and sanitary infrastructure (onebillion people live in slums) are among the most vulnerable to the harmful effects due to climate change. ${ }^{9}{ }^{17}$ However, if studies on associations between health and climate change have notably increased in the scientific literature ${ }^{20}$ since 1990, and also in the international media with an average of $4 \%$ per year, ${ }^{19}$ the review of Verner and colleagues ${ }^{20}$ underlines the need for further research on the links between climate change and health, particularly for quantitative studies ${ }^{21}$ non-communicable diseases, malnutrition and mental health. ${ }^{18} 192223$ In addition, there is also a need for further research to address the lack of knowledge about the impacts of climate change on other infectious and vector-borne diseases, particularly in slums. ${ }^{910}$

\section{Research studies: towards a holistic approach?}

Several field developments, such as Ecohealth, One Health and, more recently, Planetary Health try to address the complexity of the interconnections between ecosystems, environments and health, where climate change is considered an important driver. ${ }^{19}{ }^{20}$ Climate change and health are linked through a very wide range of mechanisms (political, environmental, social, economic and individual). This leads us to note that research groups of different fields (environmental, earth and space, health, human and social sciences, life sciences, economy and policies) carry out research studies on climate change and human health. Moreover, these research studies are achieved through various methods (quantitative, ${ }^{24}$ qualitative $^{2526}$ and mixed ${ }^{27} 28$, considering the state of knowledge, the availability of data, the analytical and spatial scale, and the determinants of health. Thus, while the importance of interdisciplinary research seems obvious, ${ }^{29-31}$ we need to inquire how the research on links between climate change and human health is holistic.

\section{Aims}

This scoping review aimed to understand how research on the links between climate change and human health in urban areas is conducted and how health is considered in this field of research.

\section{METHODOLOGY}

This study protocol has not been registered in PROSPERO because it is a scoping review. We will use the guidance document Preferred Reporting Items for Systematic Reviews and Meta-Analyses Extension for Scoping Reviews $^{32}$ to guide and to provide a checklist for this review. This scoping review will be guided by the Arskey and O'Malley ${ }^{33}$ scoping review framework and the contribution from Levac et al. ${ }^{34}$ The framework is composed of six stages:

1. Identifying the research question.

2. Identifying relevant studies.

3. Study selection.

4. Charting the data.

5. Collating, summarising and reporting the results.

6. Consultation.

\section{Review team}

The review team is composed of experienced researchers with a background in public health, environmental and urban health, climate change and urbanisation, and literature review (scoping and systematic review).

\section{Framework stage 1: identifying the research question}

In this review, the research question is as follows: how is research conducted on the links between climate change and human health in urban areas?

Subquestions are as follows:

- What are the main fields of interest considered in these research?

- What are the methods used?

- What are the determinants of health studied?

\section{Framework stage 2: identifying relevant studies} Databases and keywords

A broad range of databases will be used in the review, including PubMed (US National Library of Medicine, Bethesda, Maryland, USA) ScienceDirect (Elsevier B.V., Amsterdam, The Netherlands), Web of Science Core Collection (Thomson Reuteurs, New York, New York, USA), GreenFILE and Information Science \& Technology Abstracts (via EBSCOhost Information Services, Birmingham, Alabama, USA).

The search for keywords will be carried out using the terms "climate change" and "health" found only in the titles (and not in the abstracts and full texts) of the documents searched in the different databases. The intentional choice to limit the search strategy based on two keywords-climate change and health in the titles-is likely to lead a bias in the analysis of the findings and to discard more specifically relevant topics. Although 


\begin{tabular}{ll}
\hline Table 1 & PCC framework \\
\hline Criteria & Determinants \\
\hline P & Urban population (all ages, all ethnicities, all health conditions, all socioeconomic conditions, all countries) \\
C & $\begin{array}{l}\text { Mapping the links between climate change and human health through social and environmental determinants of } \\
\text { health }\end{array}$ \\
C & $\begin{array}{l}\text { A holistic approach based on different criteria (determinants of health, methods, fields of expertise the research } \\
\text { team and the origin of the authors' country) }\end{array}$ \\
\hline
\end{tabular}

PCC, population-concept-context.

the scoping review aimed to analyse the links between climate change and human health in urban areas, the term 'urban' is not among the keywords searched in the titles so as not to reduce the number of studies to be analysed. However, studies in urban areas will be filtered by applying the eligibility criteria of framework stage 3 .

We will use the Boolean term AND to separate the two keywords. The search strategy will be tested and adjusted with a pilot searching by the review team. The study search will be limited from 1 January 1990 to 31 July 2019. The start date refers to the publication of the First IPCC Assessment Report that underlined the importance of climate change and its impacts on society.

\section{Other searches}

We will check the reference list of included studies. We will also search manually in Google Scholar, and experts of the field will be contacted to identify potential additional publications.

\section{Eligibility criteria}

The population-concept-context framework has been used to determine the eligibility of the proposed research question, as illustrated in table 1.

\section{Data collection and extraction}

The search results will be imported to Zotero (software to collect, organise, cite and share research), and duplicates will be removed. The selected studies will be screened against the eligibility criteria through web and mobile app: Rayyan QCRI. ${ }^{35}$

\section{Framework stage 3: study selection}

The review team met to discuss decisions surrounding the study inclusion and exclusion criteria (table 2) at the beginning of the scope process.

Primarily, we will pilot testing eligibility criteria on a sample of 100 studies to train reviewers and increase the consistency of the selection. ${ }^{36}$ Then, we will select studies in a two-step process: (1) first, three reviewers will screen independently titles and abstracts; (2) then, potential relevant full texts will be retrieved and screened. Any discrepancy between reviewers will be resolved through discussion and consensus.

We emphasise that reviewers will meet at the initial, midpoint and final phases of the abstract review process to discuss the challenges or uncertainties related to the strategy selection of the study and to go back and refine the search strategy, if needed. We will adjust the selection criteria to obtain a good level of agreement between raters (Cohen's kappa coefficient of $>75 \%$ ) before conducting the final selection. In case of any modifications, the eligibility criteria will be updated, and any discrepancy will be discussed and resolved. Preferred Reporting Items for Systematic Reviews and Meta-Analyses flow diagram will report final numbers once the review is completed.

\section{Framework stage 4: charting the data}

A data charting form will be developed for the extraction of data from eligible articles, including author, date of publication, type of study and characteristics of the study (eg, geographical setting, geographical scale, target population, methods, data and indicators used in the study). We will also examine climate change policies

Table 2 Inclusion and exclusion criteria

\begin{tabular}{ll} 
Inclusion criteria & Exclusion criteria \\
\hline Focus on climate change and human health in urban areas. & Non-human health studies. \\
Peer-reviewed articles (case study, conceptual models and & Circumpolar and indigenous peoples. \\
frameworks) in English. & Economic studies based on food security and energy \\
No restriction to country. & system. \\
Urban population. & Types of texts: books, chapters books, commentaries, \\
& correspondences, editorial, opinion articles, overviews, \\
& proceedings, reports and PhD thesis. \\
& Pre-1990 publications. \\
& Review articles.
\end{tabular}


(adaptation and mitigation) considered, human health outcomes (direct and indirect effects), determinants of health studied, research team information (research fields and origin of the authors' country), health inequalities considered, as well as the limitations, gaps and challenges identified. This set of information will allow us to estimate the holistic nature of the research under analysis in the next framework stage.

Two reviewers will conduct data extraction independently. If necessary, a third reviewer will be involved if there is no agreement on the selection of articles. In addition, a qualitative thematic analysis approach will be undertaken to characterise included articles by categorising and presenting the key themes of the data.

\section{Framework stage 5: collating, summarising and reporting the results}

Scoping reviews aim to examine the extent, range and nature of the research theme on a broad range of aspects of studies and to provide a narrative synthesis. ${ }^{33}$ Quantitative data will be reported using descriptive numerical summary analysis. We will use a qualitative synthesis to describe how research is conducted and the main research fields addressed.

However, potentially relevant results from studies on climate change and human health may be overlooked because of the keywords used in the search strategy.

We will present the summary characteristics of the publications reported in tables to support the narrative synthesis. One key point of the research consists of assessing the holistic approach degree of the studies using a qualitative ranking scale (low, medium or high) according to criteria related, in particular, to social and environmental determinants of health. On another hand, we aimed to develop a new conceptual framework to support system thinking methods helping us to better consider simultaneously health, well-being, social and environmental outcomes. We will discuss the findings and their implications for future research in urban planning policies and practices.

We will not conduct a quality assessment of included studies because the aims of our review were not to provide evidence data or causality relationships, ${ }^{37}$ but rather to clarify concepts and trends about climate change and human health in urban areas. We will extract and report study design and methods of included studies that will provide some information on methodological issues.

\section{Framework stage 6: consultation}

A specific committee will be formed to suggest additional information and to discuss the findings of the scoping review proposed here. Therefore, public health professionals (Regional Health Observatory, Regional Health Agency, Rennes City Hall), professors and experts from the National French School of Public Health, and urban planning professionals (Rennes Urban Planning Agency, Practitioners from Rennes municipality) will be consulted. The consultation will be conducted using a questionnaire or through interviews with the technical and scientific committees.

\section{Patient and public involvement}

Patients and the public were not involved in the development of this protocol.

\section{DISCUSSION}

This review will provide a map of how a holistic approach is adopted or not to consider research on climate change and human health. It aimed to propose a new framework to better consider health, well-being, social and environmental outcomes through a large panel of determinants of health. In line with the planetary health vision, the findings of this work would help move forward scientific practices to more integrative approaches in environmental and human health fields of development.

However, some weaknesses can be highlighted in the search strategy. In particular, the search will be limited to English-language studies, which probably leaves out research that would be relevant to the analysis. Besides, the intentional choice to limit the search strategy based on two keywords-climate change and health in the titles-may lead to bias in the analysis. Indeed, some relevant studies may not mention these specific terms in their title (but other related terms such as risk, resilience, hazard and vulnerable people) but may nevertheless have content related to the topic that would have contributed to the discussion.

\section{Ethics and dissemination}

The findings of the scoping review will be disseminated through publication in a peer-reviewed journal, presentations at conferences relevant to the field of this research, as well as presentations to relevant stakeholders.

Contributors AR-LG, HPB and M-FT wrote the original protocol and drafted the paper. J-PR supervised this work. J-PR and CD revised the paper. All authors critically reviewed and approved the final version of the protocol.

Funding This work was funded by the Brittany Regional Health Agency (Agence Régionale de Santé Bretagne, France) under the ALADINS project (Aide à LA Décision pour caractériser et lutter contre les INégalités de Santé dans les territoires bretons).

Competing interests None declared.

Patient and public involvement Patients and/or the public were not involved in the design, conduct, reporting or dissemination plans of this research.

Patient consent for publication Not required.

Provenance and peer review Not commissioned; externally peer reviewed.

Open access This is an open access article distributed in accordance with the Creative Commons Attribution Non Commercial (CC BY-NC 4.0) license, which permits others to distribute, remix, adapt, build upon this work non-commercially, and license their derivative works on different terms, provided the original work is properly cited, appropriate credit is given, any changes made indicated, and the use is non-commercial. See: http://creativecommons.org/licenses/by-nc/4.0/.

ORCID iD

Hiago Pereira Barbosa http://orcid.org/0000-0002-8628-4491 


\section{REFERENCES}

1 Whitmee S, Haines A, Beyrer C, et al. Safeguarding human health in the Anthropocene epoch: report of the Rockefeller Foundation-Lancet Commission on planetary health. The Lancet 2015;386:1973-2028.

2 OECD. Towards Sustainable Household Consumption?: Trends and Policies in OECD Countries. OECD, 2002.

3 Thrifty ED. Green or frugal: reflections on sustainable consumption in a changing economic climate. Geoforum 2011;42:550-7.

4 Haines A, Scheelbeek P, Abbasi K. Challenges for health in the Anthropocene epoch. BMJ 2019;364:1460.

5 United Nations,, Department of Economic and Social Affairs,, Population Division. World urbanization prospects: the 2018 revision (ST/ESA/SER.A/420. New York, USA: United Nations, 2019. https://population.un.org/wup/Publications/Files/WUP2018-Report. pdf

6 Kingsley M, Ontario E, EcoHealth Ontario. Commentary - Climate change, health and green space co-benefits. Health Promot Chronic Dis Prev Can 2019;39:131-5.

7 Sverdlik A. III-Health and poverty: a literature review on health in informal settlements. Environ Urban 2011;23:123-55.

8 Bambrick H, Moncada S, Briguglio M. Climate change and health vulnerability in informal urban settlements in the Ethiopian Rift Valley. Environmental Research Letters 2015;10:054014.

9 Ezeh A, Oyebode O, Satterthwaite D, et al. The history, geography, and sociology of slums and the health problems of people who live in slums. Lancet 2017;389:547-58.

10 Lilford RJ, Oyebode O, Satterthwaite D, et al. Improving the health and welfare of people who live in slums. Lancet 2017;389:559-70.

11 Intergovernmental panel on climate change. Summary for policymakers. Cambridge, United Kingdom and New York, NY, USA: Cambridge University Press, 2013.

12 Revi A, Satterthwaite DE, Aragón-Durand F, et al. Urban areas. Cambridge, United Kingdom and New York, NY, USA: Cambridge University Press, 2014.

13 Smith KR, Woodward D, Campbell-Lendrum D, et al. Human health: impacts, adaptation, and co-benefits. Cambridge, United Kingdom and New York, NY, USA: Cambridge University Press, 2014.

14 Besancenot J-P. Changement climatique et santé. Environnement Risques Santé 14, 2015: 394-414.

15 World Health Organization. COP24 special report health and climate change, 2018. Available: https://apps.who.int/iris/bitstream/handle/ 10665/276405/9789241514972-eng.pdf

16 European Academies Science Advisory Council. The imperative of climate action to protect human health in Europe. Brussels, Belgium, 2019. Available: https://easac.eu/fileadmin/PDF_s/reports_ statements/Climate_Change_and_Health/EASAC_Report_No_38_ Climate_Change_and_Health.pdf

$17 \mathrm{Kim} \mathrm{K}-\mathrm{H}, \mathrm{Kabir} \mathrm{E}$, Ara Jahan S. A review of the consequences of global climate change on human health. J Environ Sci Health C Environ Carcinog Ecotoxicol Rev 2014;32:299-318.

18 Berry HL, Bowen K, Kjellstrom T. Climate change and mental health: a causal pathways framework. Int J Public Health 2010;55:123-32.

19 Watts N, Amann M, Arnell N, et al. The 2018 report of the Lancet countdown on health and climate change: shaping the health of nations for centuries to come. Lancet 2018;392:2479-514.
20 Verner G, Schütte S, Knop J, et al. Health in climate change research from 1990 to 2014: positive trend, but still underperforming. Glob Health Action 2016;9:30723.

21 Hosking J, Campbell-Lendrum D. How well does climate change and human health research match the demands of policymakers? A scoping review. Environ Health Perspect 2012;120:1076-82.

22 P-C W, Lee C-C. Confronting the Health-Related Challenges of Climate Change: Nursing Education for the Future]. Hu Li Za Zhi 2016;63:5-12.

23 Berry P, Enright PM, Shumake-Guillemot J, et al. Assessing health vulnerabilities and adaptation to climate change: a review of international progress. Int J Environ Res Public Health 2018;15:2626-3.

24 Grasso M, Manera M, Chiabai A, et al. The health effects of climate change: a survey of recent quantitative research. Int J Environ Res Public Health 2012;9:1523-47.

25 Cardwell FS, Elliott SJ. Making the links: do we connect climate change with health? A qualitative case study from Canada. BMC Public Health 2013;13:208.

26 Herrmann A, Sauerborn R. General Practitioners' Perceptions of Heat Health Impacts on the Elderly in the Face of Climate Change-A Qualitative Study in Baden-Württemberg, Germany. Int J Environ Res Public Health 2018;15:843-3.

27 Valois $\mathrm{P}$, Blouin P, Ouellet $\mathrm{C}$, et al. The health impacts of climate change: a continuing medical education needs assessment framework. J Contin Educ Health Prof 2016;36:218-25.

28 Toan DTT, Kien VD, Bao Giang K, et al. Perceptions of climate change and its impact on human health: an integrated quantitative and qualitative approach. Glob Health Action 2014;7:23025.

29 Haines A, Kovats RS, Campbell-Lendrum D, et al. Climate change and human health: impacts, vulnerability, and mitigation. The Lancet 2006;367:2101-9.

30 Rosenthal JK, Sclar ED, Kinney PL, et al. Links between the built environment, climate and population health: interdisciplinary environmental change research in New York City. Ann Acad Med Singap 2007;36:834-46.

31 Marselle MR, Stadler J, Korn H, et al. Biodiversity and Health in the Face of Climate Change: Perspectives for Science, Policy and Practice. In: Marselle MR, Stadler J, Korn H, et al, eds. Biodiversity and Health in the Face of Climate Change. Cham: Springer International Publishing, 2019: 451-72.

32 Tricco AC, Lillie E, Zarin W, et al. PRISMA extension for scoping reviews (PRISMA-ScR): checklist and explanation. Ann Intern Med 2018;169:467.

33 Arksey H, O'Malley L. Scoping studies: towards a methodological framework. Int J Soc Res Methodol 2005;8:19-32.

34 Levac D, Colquhoun H, O'Brien KK. Scoping studies: advancing the methodology. Implement Sci 2010;5:69.

35 Ouzzani M, Hammady H, Fedorowicz Z, et al. Rayyan-a web and mobile APP for systematic reviews. Syst Rev 2016;5:210.

36 Lefebvre C, Glanville J, Briscoe S, et al. Chapter 4: Searching for and selecting studies. In: Cochrane Handbook for Systematic Reviews of Interventions version 6.0, 2019. https://training.cochrane.org/ handbook/current/chapter-04\#section-4-5

37 Munn Z, Peters MDJ, Stern C, et al. Systematic review or scoping review? guidance for authors when choosing between a systematic or scoping review approach. BMC Med Res Methodol 2018;18:143. 